\title{
Influencia de los factores ambientales-analíticos sobre el fenotipo de esclerosis lateral amiotrófica en un medio rural
}

\author{
Cristina González-Mingot, Inés Romagosa Sánchez-Monge, Francisco Purroy, M. José Solana-Moga, \\ Silvia Peralta-Moncusí, Claudia Lazo-Latorre, M. Pilar Gil-Villar, Lluís Brieva
}

Introducción. Existen discrepancias en los diversos estudios que intentan correlacionar los factores de riesgo de desarrollar esclerosis lateral amiotrófica (ELA) y el impacto en su pronóstico. Algunos de dichos factores son intrínsecos al medio de vida rural. Por ello, proponemos estudiar si alguno de éstos influye sobre el inicio o el pronóstico de la enfermedad en la región sanitaria de Lleida, un ámbito predominantemente rural.

Pacientes y métodos. Se recogieron y analizaron variables relacionadas con factores generales, clínicos, ambientales y de laboratorio en el momento del diagnóstico de ELA en 38 pacientes, y se relacionaron retrospectivamente con el inicio de la enfermedad y su supervivencia.

Resultados. Los antecedentes familiares de $\operatorname{ELA}(p<0,02)$ y la creatincinasa elevada $(p<0,0001)$ se asociaron a mayor supervivencia. El tabaquismo $(p<0,04)$, el trabajo físico $(p<0,03)$, la creatinina baja $(p<0,03)$ y la creatincinasa eleva$\mathrm{da}(p=0,0005)$ se asociaron a un inicio precoz de la enfermedad de modo estadísticamente significativo. La forma de inicio bulbar se relacionó significativamente con un inicio tardío de la enfermedad $(p<0,01)$. El colesterol total y la proteína $C$ reactiva no influyeron en el inicio ni en el curso de la ELA en nuestra muestra. Existe una tendencia estadísticamente no significativa a favor de que el ejercicio físico moderado se asocia con un inicio más tardío, mientras que el ejercicio intenso se asocia con un inicio precoz de la ELA.

Conclusiones. Los resultados apuntan a que existe una serie de factores que influye en el desarrollo y pronóstico de la ELA, y alguno de éstos es más prevalente en el ámbito rural, como el trabajo físico.

Palabras clave. ELA. Esclerosis lateral amiotrófica. Factores ambientales. Fenotipos. Pronóstico. Supervivencia.

\section{Introducción}

La esclerosis lateral amiotrófica (ELA) es una enfermedad neurodegenerativa de las motoneuronas de la corteza cerebral, el tronco del encéfalo y la médula espinal que provoca una parálisis muscular rápidamente progresiva [1]. El espectro de la enfermedad es amplio, y los distintos fenotipos reflejan la heterogeneidad de los síntomas y la variabilidad de su curso, con una evolución inexorablemente progresiva y un desenlace fatal.

En Europa, la incidencia se mantiene uniforme, con 1,5-2,5 casos/100.000 habitantes, y la prevalencia es de 4-6 casos/100.000 habitantes [2]. La edad media de inicio es de 60,5 años y 50,5 en los casos familiares (5-10\% del total). Es más frecuente en los hombres ( 3 a 2 respecto a las mujeres) y tiene una supervivencia media de tres años [3].

Existen diversas hipótesis sobre su etiología, pero la mayoría coincide en un posible origen multifactorial centrado en el daño oxidativo $[4,5]$. A pesar de tener bien definidos algunos factores con alto impacto sobre la supervivencia de la ELA, como el índice de masa corporal, la ventilación mecánica y la rehabilitación precoz [6,7], sigue habiendo discrepancias respecto a otros factores que se han intentado correlacionar con el pronóstico y los distintos fenotipos de la enfermedad [8]. Un ejemplo de ello es el trabajo de un grupo alemán que concluye que la elevada edad en el momento del diagnóstico, el sexo masculino y el inicio bulbar de la enfermedad tenían peor pronóstico [9].

Por otro lado, niveles elevados de creatinina [10] y bajos de creatincinasa [11] se han asociado a mayor supervivencia. A pesar de que anteriormente se propusieron los niveles altos de colesterol como factor protector en la ELA, trabajos recientes apuntan a que no modificarían el curso de la enfermedad [12]. En lo referente al tabaco se considera que, además de ser un factor de riesgo de desarrollar ELA, puede disminuir la supervivencia en las mujeres [13]. Un metaanálisis inglés apunta que el riesgo de ELA aumenta en los expuestos a pesticidas y los agricultores [14]. Además, los metales pesados [15]
Hospital Universitari Arnau de Vilanova (C. González-Mingot, F. Purroy, M.J. Solana-Moga, S. Peralta-Moncusí, C. Lazo-Latorre, M.P. Gil-Villar, LI. Brieva). Universitat de Lleida (I. Romagosa SánchezMonge). Lleida, España.

Correspondencia: Dra. Cristina González Mingot. Servicio de Neurología. Hospital Arnau de Vilanova. Avda. Alcalde Rovira Roure, 80. E-25198 Lleida.

E-mail:

crismingot@hotmail.com

Aceptado tras revisión externa: 06.03.17.

Cómo citar este artículo: González-Mingot C, Romagosa Sánchez-Monge I, Purroy F, SolanaMoga MJ, Peralta-Moncusí S, LazoLatorre C, et al. Influencia de los factores ambientales-analíticos sobre el fenotipo de esclerosis lateral amiotrófica en un medio rural. Rev Neurol 2017; 65: 203-8.

(c) 2017 Revista de Neurología 
Figura 1. Factores generales en función de la edad de inicio y supervivencia.

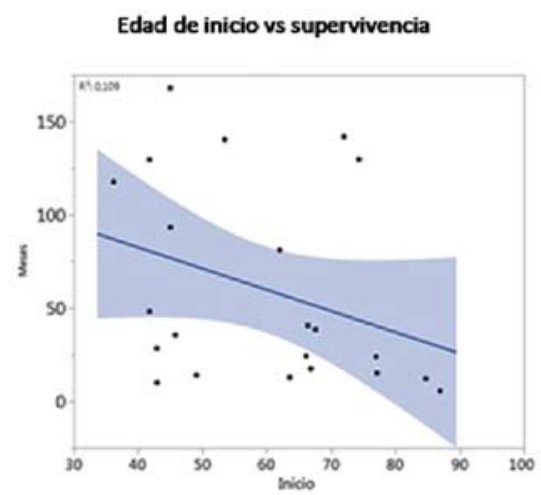

$r^{2}=0,11 \rightarrow p=0,13$

Antecedentes familiares vs inicio y supervivencia

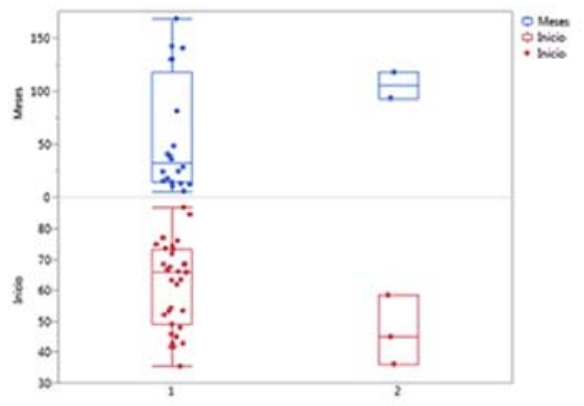

INICIO: $p=0,06$

No: $35 \quad 61,99 \pm 13,52$

Si: $3 \quad 46,58 \pm 11,27$

SUPERVIVENCIA: $p=0,02$

No: $20 \quad 55,91 \pm 54,15$

Sí: $2105,5 \pm 17,25$

Sexo vs inicio y supervivencia

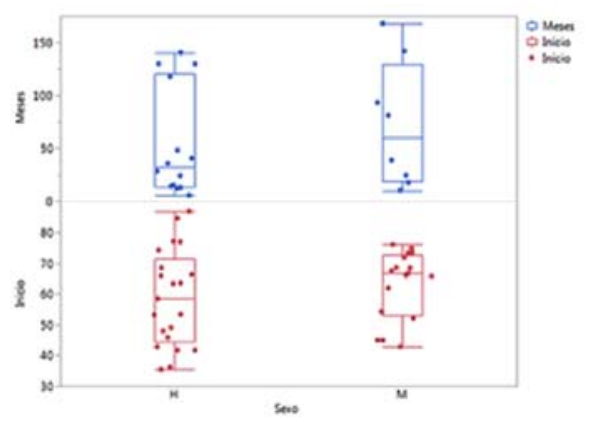

INICIO: $p=0,15$

H: $2153,8 \pm 15,7$

M: $17 \quad 63,2 \pm 11,1$

SUPERVIVENCIA: $p=0,24$

H: $14 \quad 53,87 \pm 51,14$

M: $\quad 8 \quad 71,87 \pm 59,57$

y otros factores ambientales, como el deporte intenso [16], se han considerado posibles factores de riesgo, sin conocerse aún su relación con el pronóstico de la enfermedad.

Dado que el medio de vida rural conlleva, en una gran parte de los casos, una mayor actividad física a nivel laboral, una superior exposición a pesticidas y una dieta concreta, quisimos estudiar estos y otros factores en nuestra muestra, buscando si alguno de ellos influye en el inicio y el pronóstico de la enfermedad.

\section{Pacientes y métodos}

Se realizó un estudio observacional de 38 pacientes con ELA. Se analizó una serie de factores en el momento del diagnóstico y se relacionaron con la edad de inicio y su supervivencia. Los datos provienen de las historias clínicas de pacientes de la unidad de ELA del Hospital Arnau de Vilanova de Lleida, previa firma del consentimiento informado. Para el análisis estadístico se utilizó el programa JMP12, del SAS Institute.

Los factores estudiados fueron los siguientes: generales (edad en el momento del diagnóstico, sexo, antecedentes familiares), clínicos (afectación inicial de la motoneurona superior o inferior, región de inicio bulbar o espinal), ambientales (población de residencia, profesión, ejercicio físico, exposición a pesticidas o metales pesados y tabaquismo) y de laboratorio (colesterol total, creatinina y proteína $\mathrm{C}$ reactiva en el momento del diagnóstico).

\section{Resultados}

\section{Demografía básica}

La muestra constaba de 21 hombres $(55,3 \%)$ y 17 mujeres $(44,7 \%)$. La edad media de inicio fue de $60,8 \pm 13,9$ años, y la duración de la enfermedad en los 14 pacientes fallecidos fue de $23,4 \pm 13,1$ meses. Un 18,4\% sobrevivió menos de 24 meses; un 15,8\%, entre 24-48 meses, y un 23,7\%, más de 48 meses, incluyendo los vivos que llevaban en el momento del análisis más de 48 meses de evolución. El 42,1\% restante estaba vivo, con una supervivencia menor de 48 meses (Tabla).

\section{Factores generales}

El inicio tardío de la enfermedad se asoció a peor pronóstico de forma no significativa $(p=0,13)$. Los antecedentes familiares se asociaron a inicio precoz de forma casi significativa $(p=0,064)$ y a mayor supervivencia $(p=0,02)$. Aunque de forma no significativa, los varones tuvieron un inicio precoz de la enfermedad y una menor supervivencia (Fig. 1).

\section{Factores clínicos}

Los pacientes con inicio bulbar presentaron la enfermedad en edades más tardías de forma estadísticamente significativa $(p<0,01)$, pero no se detectaron diferencias en la supervivencia. No se hallaron diferencias entre los pacientes que presentaban un 
predominio de la motoneurona superior, la motoneurona inferior o ambas en el diagnóstico (Fig. 2).

\section{Factores ambientales}

Encontramos una tendencia no significativa respecto a una mayor frecuencia de ELA en el medio rural que en la ciudad ( $p=0,135)$, aunque no se encontraron diferencias en la edad de inicio y la supervivencia de estos grupos. Sin ser estadísticamente significativo, parece que el ejercicio libre (o no laboral) moderado retrasa el inicio de la enfermedad, y el intenso lo adelanta. La actividad laboral física se asoció a inicio precoz de la ELA $(p<0,03)$ (Fig. 3).

La exposición reiterada a pesticidas y metales pesados, y los trabajos con alta exposición a químicos (agricultores, limpieza...) no alteraron la edad de inicio de la enfermedad.

El tabaquismo se asoció a inicio precoz de la enfermedad $(p<0,04)$, pero no alteró su pronóstico.

\section{Factores analíticos}

Valores bajos de creatinina se asociaron a inicio precoz de la ELA $(p<0,03)$ y no alteraron su pronóstico. Valores elevados de creatincinasa se asociaron a inicio precoz $(p=0,0005)$ y a mejor pronóstico $(p=$ $0,0001)$. El colesterol total y la proteína $C$ reactiva no alteraron el inicio ni el pronóstico de ELA (Fig. 4).

\section{Discusión}

Cabe destacar que el tamaño muestral estudiado es reducido, en parte debido a la baja incidencia de la ELA, lo que limita la capacidad de detectar asociaciones significativas. El inicio tardío de la enfermedad se asoció de forma no significativa a menor supervivencia, de la misma manera que en estudios anteriores [9].

En línea con publicaciones previas, los antecedentes familiares se asociaron a inicio precoz y mejor pronóstico de ELA [17]. Respecto a las discrepancias existentes en cuanto al diferente pronóstico en función del sexo $[8,9]$, en nuestra muestra no se observaron diferencias. La motoneurona predominante inicial no alteró la supervivencia en nuestro caso. En cuanto a la región de inicio, se vio que los pacientes con ELA bulbar iniciaron la enfermedad en edades más tardías que los de inicio espinal.

El tabaco tuvo un impacto en el inicio de la ELA, pero no en su supervivencia, a diferencia de otros estudios, en los que se relacionaba con un peor pronóstico [13]. En la misma línea que estudios previos
Figura 2. Factores clínicos en función de la edad de inicio y supervivencia.
Inicio bulbar/no bulbar vs inicio y supervivencia

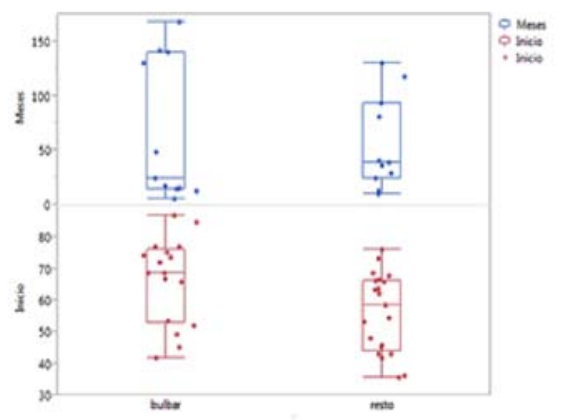

MNS o MNI/MNS + MNI vs inicio y supervivencia

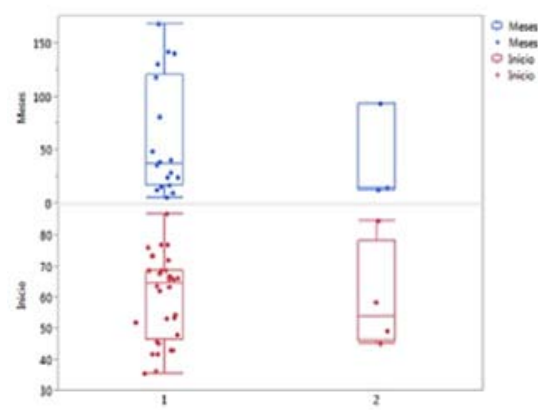

INICIO: $\mathrm{p}=0,01$

Bulbar: $1766,60 \pm 13,59$

No bulbar: $21 \quad 56,05 \pm 12,49$

SUPERVIVENCIA: $p=0,34$

Bulbar: $1165,17 \pm 64,84$

No bulbar: $1155,67 \pm 42,36$
INICIO: $p=0,47$

MNS/MNI: $3260,08 \pm 13,63$

MNS + MNI: $4 \quad 59,35 \pm 17,86$

SUPERVIVENCIA: $p=0,27$

MNS/MNI: $18 \quad 59,99 \pm 54,29$

MNS + MNI $3 \quad 39,84 \pm 46,30$
Tabla. Presentación de la muestra.

\begin{tabular}{|c|c|c|c|c|c|}
\hline & Estado & $n$ & $\begin{array}{l}\text { Edad de inicio } \\
\quad(\text { años })^{a}\end{array}$ & $\begin{array}{l}\text { Evolución } \\
\text { (meses) }^{a}\end{array}$ & $\begin{array}{c}\text { Supervivencia } \\
\text { (meses) }^{a}\end{array}$ \\
\hline \multirow{5}{*}{ Mujeres } & Vivas & 13 & $64,0 \pm 11,3$ & $52,9 \pm 53,2$ & \\
\hline & $<48$ meses & 9 & $67,5 \pm 8,8$ & $22,6 \pm 16,2$ & \\
\hline & $>48$ meses & 4 & $56,0 \pm 13,3$ & $121,1 \pm 40,9$ & \\
\hline & Fallecidas & 4 & $60,9 \pm 12,0$ & & $22,6 \pm 12,1$ \\
\hline & Total & 17 & $63,2 \pm 11,1$ & & \\
\hline \multirow{5}{*}{ Hombres } & Vivos & 11 & $54,4 \pm 13,1$ & $58,6 \pm 56,9$ & \\
\hline & $<48$ meses & 7 & $56,1 \pm 11,6$ & $18,1 \pm 9,8$ & \\
\hline & $>48$ meses & 4 & $51,4 \pm 16,9$ & $129,4 \pm 9,3$ & \\
\hline & Fallecidos & 10 & $63,5 \pm 17,6$ & & $23,7 \pm 14,1$ \\
\hline & Total & 21 & $58,8 \pm 15,7$ & & \\
\hline \multicolumn{2}{|c|}{ Total general } & 38 & $60,8 \pm 13,9$ & $55,5 \pm 53,8$ & $23,4 \pm 13,1$ \\
\hline
\end{tabular}

a Media £ desviación estándar. 
Figura 3. Factores ambientales en función de la edad de inicio y supervivencia.
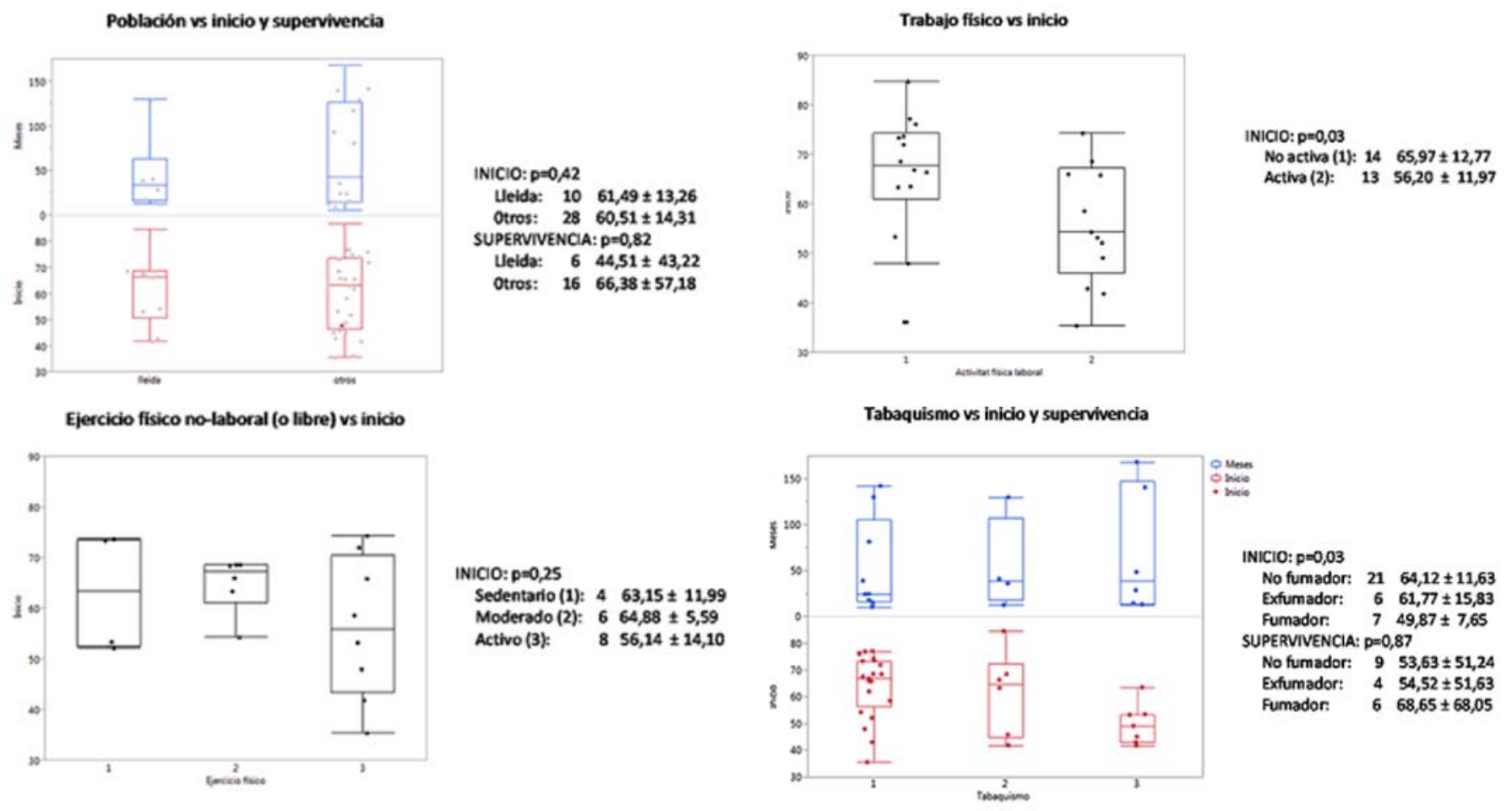

que encuentran relación entre la ELA y la zona de residencia [14], hallamos una mayor prevalencia de la ELA en el medio rural, pero que no resulta estadísticamente significativa.

En nuestra muestra, el trabajo físico se asoció de forma estadísticamente significativa a un inicio precoz de la ELA. Por otro lado, el ejercicio no laboral intenso parece adelantar el inicio de la enfermedad, mientras que el moderado lo retrasaría. Nuestros datos vendrían a confirmar los obtenidos por Huisman et al, quienes demostraron en un amplio estudio una mayor incidencia de ELA en pacientes que realizaban ejercicio no laboral y una tendencia no significativa en los que hacían un trabajo físico [16].

Aunque no hemos podido confirmar, como en trabajos previos, que niveles elevados de creatinina se relacionan con un mejor pronóstico [10], nuestros resultados muestran que se asociaban a inicio tardío de la enfermedad. Teniendo en cuenta que la creatinina tiene cierta correlación con la masa muscular y el índice de masa corporal, y que éste es un factor pronóstico de ELA [18], resulta fácil entender que valores elevados de creatinina se asocien a mayor índice de masa corporal y, por tanto, a mejor pronóstico.

Valores elevados de creatincinasa se asociaron a un inicio precoz de la enfermedad y, en contraposición a estudios anteriores [11], a una mayor supervivencia. La explicación que proponemos es que los pacientes que tienen la creatincinasa elevada suelen ser más jóvenes (ya que la creatincinasa se asocia con una mayor masa muscular) y, por tanto, tienen una supervivencia más elevada [11].

El colesterol total, en la misma línea de trabajos recientes [12], no modificó el curso de la enfermedad en nuestros pacientes. Creemos que estos resultados se deben a que los niveles de colesterol total no se correlacionan con el índice de masa corporal (que sí influye sobre el pronóstico) [19]. El valor de proteína $C$ reactiva tampoco mostró ninguna diferencia sobre el inicio ni el pronóstico de la enfermedad.

En conclusión, aunque el tamaño reducido de la muestra hace que seamos cautelosos en las conclusiones, hemos encontrado algunas asociaciones sig- 
Figura 4. Factores analíticos en función de la edad de inicio y supervivencia.
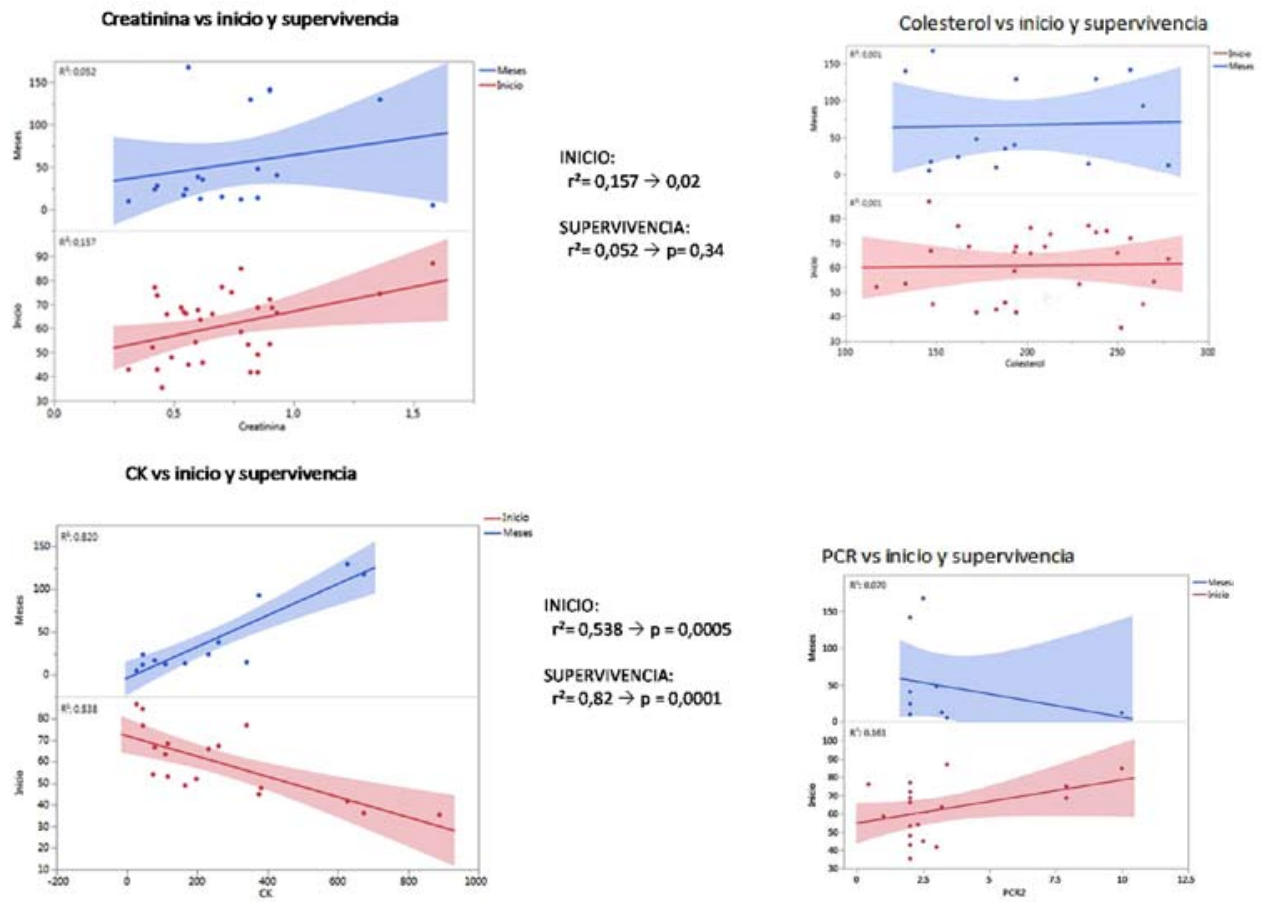

nificativas que, en la mayoría de casos, siguen la misma línea que investigaciones previas.

Se dibuja un fenotipo de pacientes cuya supervivencia es mayor que el resto, independientemente de la actitud terapéutica que adoptemos con ellos. Parece ser que hay una serie de factores que variarían la supervivencia de ELA y otros que podrían predisponer a su desarrollo.

En nuestra muestra, observamos una mayor prevalencia de ELA en las zonas rurales que en la ciudad. Además, se relacionan con una mayor supervivencia los antecedentes familiares y los valores elevados de creatincinasa. Por otro lado, se asociaron con un inicio precoz de la ELA el tabaquismo, el trabajo físico, las formas familiares, y los niveles bajos de creatinina y altos de creatincinasa. Las formas de inicio bulbar se relacionaron con un inicio tardío. El colesterol total y la proteína $C$ reactiva no alteraron el pronóstico de la ELA. Además, parece que el ejercicio físico intenso podría asociarse a un inicio precoz de la ELA, mientras que el ejercicio moderado retrasaría su inicio.

De esta forma, podemos concluir que la vida en el medio rural se relaciona con ciertos factores que tienen influencia en el desarrollo y en el pronóstico de la ELA.

Bibliografía

1. Andrews J. Amyotrophic lateral sclerosis: clinical management and research update. Curr Neurol Neurosci 2009; 9: 59-68.

2. Logroscino G, Traynor BJ, Hardiman O, Chiò A, Mitchell D, Swingler R, et al. Incidence of amyotrophic lateral sclerosis in Europe. J Neurol Neurosurg Psychiatry 2009; 81: 385-90.

3. Povedano M, Farrero E, Virgili N. Diagnòstic i tractament de l'esclerosi lateral amiotròfica. Guies mèdiques de la Societat Catalana de Neurologia. 2 ed. Barcelona: SCN; 2011.

4. Agar J, Durham H. Relevance of oxidative injury in the pathogenesis of motor neuron diseases. Amyotroph Lateral Scler Other Motor Neuron Disord 2003; 4: 232-42.

5. Vucic S, Kiernan MC. Pathophysiology of neurodegeneration in familial amyotrophic lateral sclerosis. Curr Mol Med 2009; 9: 255-72.

6. Dupuis L, Oudart H, Rene F, González de Aguilar JL, Loeffler JP. Evidence for defective energy homeostasis in amyotrophic lateral sclerosis: benefit of a high-energy diet in a transgenic mouse model. Proc Natl Acad Sci U S A 2004; 101: 11159-64.

7. Brownlee A, Palovcak M. The role of augmentative communication devices in the medical management of ALS. Neurorehabilitation 2007; 22: 445-50.

8. Rothstein JD. Current hypotheses for the underlying biology of amyotrophic lateral sclerosis. Ann Neurol 2009; 65 (Suppl 1): S3-9.

9. Wolf J, Safer A, Wöhrle JC, Palm F, Nix WA, Maschke M, et al. Factors predicting survival in ALS patients - data from a 
population-based registry in Rhineland-Palatinate, Germany. Neuroepidemiology 2015; 44: 149-55.

10. Chiò A, Calvo A, Bovio G, Canosa A, Bertuzzo D, Galmozzi F, et al. Amyotrophic lateral sclerosis outcome measures and the role of albumin and creatinine: a population-based study. JAMA Neurol 2014; 71: 1134-42.

11. Gibson SB, Kasarskis EJ, Hu N, Pulst SM, Mendiondo MS, Matthews DE, et al. Relationship of creatine kinase to body composition, disease state, and longevity in ALS. Amyotroph Lateral Scler Frontotemporal Degener 2015; 16: 473-7.

12. Rafiq MK, Lee E, Bradburn M, Mcdermott CJ, Shaw PJ. Effect of lipid profile on prognosis in the patients with amyotrophic lateral sclerosis: insights from the olesoxime clinical trial. Amyotroph Lateral Scler Frontotemporal Degener 2015; 16 478-84.

13. Alonso A, Logroscino G, Jick SS, Hernán MA. Association of smoking with amyotrophic lateral sclerosis risk and survival in men and women: a prospective study. BMC Neurol 2010; 10: 6 .

14. Kang H, Cha ES, Choi GJ, Lee WJ. Amyotrophic lateral sclerosis and agricultural environments: a systematic review. J Korean Med Sci 2014; 29: 1610-7.

15. Johnson FO, Atchison W. The role of environmental mercury, lead and pesticide exposure in development of amyotrophic lateral sclerosis. Neurotoxicology 2009; 30: 761-5.

16. Huisman M, Seelen M, Jong SW, Dorresteijn KR, Van Doormaal P, Van der Kooi A, et al. Lifetime physical activity and the risk of amyotrophic lateral sclerosis. J Neurol Neurosurg Psychiatry 2013; 84: 976-81.

17. Ticozzi N, Tiloca C, Morelli C, Colombrita C, Poletti B, Doretti B, et al. Genetics of familial amyotrophic lateral sclerosis. Arch Ital Biol 2011; 149: 65-82.

18. Pupillo E, Messina P, Logroscino G, Beghi E. Long-term survival in amyotrophic lateral sclerosis: a population-based study. Ann Neurol 2014; 75: 287-97.

19. Wietlisbach V, Marques-Vidal P, Kuulasmaa K, Karvanen J, Paccaud F. The relation of body mass index and abdominal adiposity with dyslipidaemia in 27 general populations of the WHO MONICA Project. Nutr Metab Cardiovasc Dis 2013; 23 : 432-42.

Environmental-analytical risk factors influence on the phenotype of amyotrophic lateral sclerosis in a rural setting

Introduction. There are discrepancies in the different studies that attempt to correlate the risk factors of developing amyotrophic lateral sclerosis (ALS) and the impact on their prognosis. Some of these factors are intrinsic to the rural livelihood. Therefore, we propose to study if any of these influence the onset and/or prognosis of the disease in the health region of Lleida, a predominantly rural area.

Patients and methods. Variables related to general factors, clinical, environmental and laboratory were collected and analyzed at the time of diagnosis of ALS in 38 patients and were retrospectively related to the onset of the disease and its survival.

Results. The family history of ALS $(p<0.02)$ and elevated CK $(p<0.0001)$ were associated with increased survival. Smoking $(p<0.04)$, physical work $(p<0.03)$, low creatinine $(p<0.03)$, elevated CK $(p=0.0005)$ were associated with an early onset of the disease. The bulbar onset form was significantly related to a late onset of the disease $(p<0,01)$. Total cholesterol and PCR did not influence the onset or course of ALS. There is a non-significant trend at statistical level in favor of moderate physical exercise being associated with a later onset, while intense exercise at an early onset of ALS.

Conclusions. The results suggest that there are a number of factors that influence the development and prognosis of ALS, some of which are more prevalent in rural areas, such as physical work.

Key words. ALS. Amyotrophic lateral sclerosis. Environmental factors. Phenotypes. Prognosis. Survival. 